This item was submitted to Loughborough's Research Repository by the author.

Items in Figshare are protected by copyright, with all rights reserved, unless otherwise indicated.

\title{
Duality and exact results for conductivity of 2D isotropic heterophase systems in magnetic field
}

\author{
PLEASE CITE THE PUBLISHED VERSION \\ LICENCE
}

CC BY-NC-ND 4.0

REPOSITORY RECORD

Bulgadaev, S.A., and F.V. Kusmartsev. 2019. "Duality and Exact Results for Conductivity of 2D Isotropic Heterophase Systems in Magnetic Field". figshare. https://hdl.handle.net/2134/1249. 


\title{
DUALITY AND EXACT RESULTS FOR CONDUCTIVITY OF 2D ISOTROPIC HETEROPHASE SYSTEMS IN MAGNETIC FIELD
}

\author{
S.A.Bulgadaev ${ }^{1}$, F.V.Kusmartsev ${ }^{2}$ \\ Landau Institute for Theoretical Physics, Chernogolovka, Russia, 142432 \\ Department of Physics, Loughborough University, Loughborough, LE11 3TU, UK
}

\begin{abstract}
Using a fact that the effective conductivity $\hat{\sigma}_{e}$ of $2 \mathrm{D}$ random heterophase systems in the orthogonal magnetic field is transformed under some subgroup of the linear fractional group, connected with a group of linear transformations of two conserved currents, the exact values for $\hat{\sigma}_{e}$ of isotropic heterophase systems are found. As known, for binary $(\mathrm{N}=2)$ systems a determination of exact values of both conductivities (diagonal $\sigma_{e d}$ and transverse Hall $\sigma_{e t}$ ) is possible only at equal phase concentrations and arbitrary values of partial conductivities. For heterophase $(N \geq 3)$ systems this method gives exact values of effective conductivities, when their partial conductivities belong to some hypersurfaces in the space of these partial conductivities and the phase concentrations are pairwise equal. In all these cases $\sigma_{e}$ does not depend on phase concentrations. The complete, 3-parametric, explicit transformation, connecting $\sigma_{e}$ in binary systems with a magnetic field and without it, is constructed.
\end{abstract}

PACS: 75.70.Ak, 72.80.Ng, 72.80.Tm, 73.61.-r

\section{Introduction}

The properties of the electrical transport of classical macroscopically inhomogeneous (random or regular) heterophase systems, consisting of $N(N \geq 2)$ phases with different conductivities $\sigma_{i}(i=1,2, \ldots, N)$, have had always an importance for practice [1. Last time new materials appeared which are very perspective from the high technology point of view. They often have the heterophase inhomogeneous structure on smaller scales (from microscopic till mesoscopic) and some unusual transport properties. For example, the magnetoresistance of oxide materials with a perovskite type structure becomes very large (the so called colossal magnetoresistance in such materials as manganites) 2] or grows approximately linearly with magnetic field up to very high fields (in silver chalcogenides) [3. There is an opinion that these properties take place due to inhomogeneities of these materials [3]. For this reason a calculation of the effective conductivity $\sigma_{e}$ of inhomogeneous heterophase systems without and with magnetic field at arbitrary partial conductivities and phase concentrations is very important problem. Unfortunately, the existing effective medium approximations (EMA) describes well the effective conductivity of random heterophase systems without magnetic field only for binary $(N=2)$ systems and at not too large inhomogeneities 4. For systems with $N>2$ phases without magnetic

\footnotetext{
${ }^{1}$ e-mail: bulgad@itp.ac.ru

${ }^{2}$ e-mail: F.Kusmartsev@lboro.ac.uk
} 
field [4, 5] and for systems in magnetic field 6] the EMA cannot give an explicit simple formulas convenient for description of the experimental results in a wide range of partial parameters. Such formulas can be obtained only in high magnetic field 6, 7

A situation is better in 2D systems, where a few exact results [8, 9, 10] and approximate explicit expressions based on exact duality relations (DR) have been obtained for isotropic self-dual heterophase systems without magnetic field [5, 11. But, the exact results for inhomogeneous systems in magnetic field have been obtained only for binary systems at equal phase concentrations $x_{1}=x_{2}=1 / 2$ [12, 13 (see also [14 about systems with uniform $\sigma_{t}$ ). In this letter we will show that the exact results for both diagonal $\left(\sigma_{e d}\right)$ and transverse $\left(\sigma_{e t}\right)$ effective conductivities can be obtained for isotropic self-dual heterophase systems (random as well as regular) in magnetic field $H$ and at arbitrary number of phases $N$. They generalize the known results for binary systems with magnetic field 12, 13 and for arbitrary $N$-phase systems without magnetic field 10. In particular, it will be shown that for $N>2$, contrary to the $N=2$ case, there exist the whole hyperplanes in the space of concentrations and hypersurfaces in the space of partial conductivities, on which $\sigma_{e}$ does not depend on phase concentrations. The full explicit transformation, connecting $\sigma_{e}$ in systems with a magnetic field and without it, is constructed.

\section{Conserved currents, symmetries and duality transformations in magnetic field}

We start with a brief description of some general properties of classical isotropic stationary conducting systems in the external perpendicular magnetic field $H$. Such systems are described by the following differential equations for a current $\mathbf{j}(\mathbf{r})$ and an electric field $\mathbf{e}(\mathbf{r})$ [1]

$$
\operatorname{div} \cdot \mathbf{j}(\mathbf{r})=\partial_{i} j_{i}(\mathbf{r})=0, \quad \nabla \times \mathbf{e}(\mathbf{r})=\epsilon_{i k} \partial_{i} e_{k}=0, \quad i, k=1,2
$$

and the Ohm law

$$
\mathbf{j}(\mathbf{r})=\hat{\sigma}(\mathbf{r}) \mathbf{e}(\mathbf{r}),
$$

where

$$
\hat{\sigma}=\sigma_{i k}=\sigma_{d} \delta_{i k}+\sigma_{t} \epsilon_{i k}, \quad \sigma_{d}(\mathbf{H})=\sigma_{d}(-\mathbf{H}), \quad \sigma_{t}(\mathbf{H})=-\sigma_{t}(-\mathbf{H}),
$$

All conductivities $\hat{\sigma}$ of the form (3) commute between themselves and can be treated as ordinary functions. The effective conductivity $\hat{\sigma}_{e}$ of $N$-phase random or regular symmetric systems with the partial conductivities $\sigma_{i d}, \sigma_{i t}(i=$ $1,2, \ldots, N)$ (we assume that $\sigma_{i d} \geq 0$ ) and concentrations $x_{i}$ (satisfying the nor-

malization condition $\left.\sum_{i=1}^{N} x_{i}=1\right)$ must be a symmetric function of pairs of arguments $\left(\hat{\sigma}_{i}, x_{i}\right)$ and a homogeneous (a degree 1) function of $\sigma_{d i, t i}$. For this reason it is invariant under all permutations

$$
\hat{\sigma}_{e}\left(\hat{\sigma}_{1}, x_{1}\left|\hat{\sigma}_{2}, x_{2}\right| \ldots \mid \hat{\sigma}_{N}, x_{N}\right)=\hat{\sigma}_{e}\left(P_{i j}\left(\hat{\sigma}_{1}, x_{1}\left|\hat{\sigma}_{2}, x_{2}\right| \ldots \mid \hat{\sigma}_{N}, x_{N}\right)\right),
$$


where $P_{i j}$ is the permutation of the $\mathrm{i}$-th and $\mathrm{j}$-th pairs of arguments, and can be represented in the next form

$$
\hat{\sigma}_{e}\left(\hat{\sigma}_{1}, x_{1}\left|\hat{\sigma}_{2}, x_{2}\right| \ldots \mid \hat{\sigma}_{N}, x_{N}\right)=\sigma_{s} \hat{f}\left(z_{1 d, 1 t}, x_{1}\left|z_{2 d, 2 t}, x_{2}\right| \ldots \mid z_{N d, N t}, x_{N}\right) .
$$

Here $z_{i d, i t}=\sigma_{i d, i t} / \sigma_{s}$, where $\sigma_{s}$ is some normalizing conductivity. It is convenient to choose $\sigma_{s}$ in the form symmetrical relative $\sigma_{i d, i t}$, what conserves a symmetry property of $f$.

The effective conductivity of $N$-phase systems must satisfy, together with the usual boundary conditions on boundaries between i-th and k-th phases

$$
j_{i n}=j_{k n}, \quad e_{i \|}=e_{k \|}, \quad i, k=1,2, \ldots, N,
$$

the following natural reduction and limiting requirements:

1) $\hat{\sigma}_{e}$ of $N$-phase system with $n(2 \leq n \leq N)$ equal partial conductivities must reduce to $\hat{\sigma}_{e}$ of system with $N-n+1$ phases and the concentrations of the phases with equal conductivities must add;

2 ) it has not depend on partial $\sigma_{d i, t i}$ and must reduce to the effective conductivity of $(N-1)$-phase system, if the concentration of this phase $x_{i}=0$;

3 ) it must reduce to some partial $\hat{\sigma}_{i}$, if $x_{i}=1$.

The duality relations for $\hat{\sigma}_{e}$ in magnetic field have in general a more richer and complicated structure, which generalizes the inversion structure of DR of systems without magnetic field. It was established firstly by Dykhne in [12, where an idea and a method to solve this problem have been proposed. We use these idea and method, refined later (see, for example, 13, 15] 16), in a modern form. The main observation is that in the $2 \mathrm{D}$ conducting system, described by equations $(1,2)$, really exist 2 conserved currents and two curlfree vector fields. The second partners for $\mathbf{j}$ and $\mathbf{e}$ are their conjugated fields, which satisfy the corresponding equations

$$
\tilde{j}_{i}=\epsilon_{i k} e_{k}, \quad \tilde{e}_{i}=\epsilon_{i k} j_{k}, \quad \partial_{i} \tilde{j}_{i}=0, \quad \epsilon_{i k} \partial_{i} \tilde{e}_{k}=0 .
$$

As a result one can consider linear combinations of conserved currents and curlfree electric fields

$$
\mathbf{j}=a \mathbf{j}^{\prime}+b \tilde{\mathbf{j}}^{\prime}, \quad \mathbf{e}=c \mathbf{e}^{\prime}+d \tilde{\mathbf{e}}^{\prime},
$$

where the coefficients $a, b, c, d \in R$ are arbitrary real. Futher it will be more convenient to use the complex representation for coordinates and vector fields 1

$$
z=x+i y, \quad j=j_{x}+i j_{y}, \quad e=e_{x}+i e_{y}, \quad \sigma=\sigma_{d}+i \sigma_{t} .
$$

The primed currents and electric field satisfy again the Ohm law

$$
j^{\prime}=\sigma^{\prime} e^{\prime}, \quad \sigma^{\prime}=T(\sigma)=\frac{c \sigma-i b}{-i d \sigma+a} .
$$

It follows from (7),(8) that under a linear transformation (7) a conductivity transforms under the corresponding linear fractional (LF) transformation $\mathrm{T}$ 
from (8). In the absence of magnetic field $\sigma$ is real (or, in a matrix representation, diagonal) and a transformation (8) reduces to the inversion [8, 9

$$
\sigma^{\prime}=\frac{\sigma_{0}^{2}}{\sigma}, \quad \sigma_{0}^{2}=b / c, \quad a=d=0
$$

Thus, instead of one-parametric inversion transformations in systems without magnetic field, $\sigma$ in magnetic field transforms under linear fractional transformation with 3 real parameters (since one of 4 parameters can be factored due to the fractional structure of $\mathrm{T}$ ). There are various ways to choose 3 parameters. In our treatment it will be convenient to factor $d$. This gives 3 parameters $\bar{a}=a / d, \bar{b}=b / d, \bar{c}=c / d$, determining a transformation $T$. The transformations $T$ from (8) form a subgroup of a group of all linear fractional transformations with arbitrary complex coefficients $a, b, c, d$, conserving the imaginary axis. This subgroup contains also the shift transformations for an imaginary part of $\sigma$, which correspond to $d=0, a=c$.

The transformation (8) has the following form in terms of conductivity components $\sigma_{d}$ and $\sigma_{t}$

$$
\begin{gathered}
\sigma_{d}^{\prime}=\sigma_{d} \frac{a c+b d}{\left(d \sigma_{d}\right)^{2}+\left(a+d \sigma_{t}\right)^{2}}=\bar{c} \sigma_{d} \frac{\bar{a}+\bar{b} / \bar{c}}{\left(\sigma_{d}\right)^{2}+\left(\bar{a}+\sigma_{t}\right)^{2}} \\
\sigma_{t}^{\prime}=\frac{c d \sigma_{d}^{2}+\left(a+d \sigma_{t}\right)\left(c \sigma_{t}-b\right)}{\left(d \sigma_{d}\right)^{2}+\left(a+d \sigma_{t}\right)^{2}}=\bar{c} \frac{\sigma_{d}^{2}+\left(\bar{a}+\sigma_{t}\right)\left(\sigma_{t}-b / c\right)}{\left(\sigma_{d}\right)^{2}+\left(\bar{a}+\sigma_{t}\right)^{2}} .
\end{gathered}
$$

They have rather interesting structure. Any general transformation gives nontrivial transverse part $\sigma_{t}^{\prime} \neq 0$, even if it was absent $\left(\sigma_{t}=0\right)$, while $\sigma_{d}^{\prime}$ remains always equal to 0 , if $\sigma_{d}=0$ (or $\sigma_{d}^{\prime}=0$, if $\operatorname{det} T=0$ ).

This general consideration can be applied also to the effective conductivity of inhomogeneous systems. Then one obtains the following transformation property for $\sigma_{e}$

$$
\begin{gathered}
\sigma_{e}\left(\left\{\sigma_{i}\right\},\left\{x_{i}\right\}\right)=T\left(\sigma_{e}\left(T\left(\left\{\sigma_{i}\right\}\right),\left\{x_{i}\right\}\right)\right), \\
\left.T^{-1}\left(\sigma_{e}\left(\left\{\sigma_{i}\right\},\left\{x_{i}\right\}\right)\right)=\sigma_{e}\left(T\left(\left\{\sigma_{i}\right\}\right),\left\{x_{i}\right\}\right)\right) .
\end{gathered}
$$

The relation (11) means that $\sigma_{e}$ transforms under conjugated representation $\left(\sim T^{-1}\right)$ of linear fractional transformations and its change as a function of its arguments can be completely compensated by the same transformation of it itself (this form takes place for the dual transformations connected with symmetry transformations satisfying the condition $T \sim T^{-1}$ and for them is equivalent to the usual case from [12]). Note also that the transformations (11) does not depend and does not act on concentrations. Following a tradition, we will call such transformations the duality transformations (DT) and such relations the duality relations (DR). An existence of the such exact duality relations is very important property of two-dimensional inhomogeneous systems. For example, they can be used for the obtaining the exact values of effective conductivity. For this one needs the transformations, which action on the set of partial conductivities, can be compensated by symmetries of the effective conductivity as 
a function of its partial conductivities and phase concentrations. It means that the set of all partial parameters and concentrations must be invariant under some joint action of the DT and symmetry operations, i.e. it must be their fixed point $(\mathrm{FP})$. At this set of partial parameters $\left(\left\{\sigma *_{i}\right\},\left\{x *_{i}\right\}\right)$ one obtains for $\sigma_{e}$ the exact equation

$$
\sigma_{e}\left(\left\{\sigma *_{i}\right\},\left\{x *_{i}\right\}\right)=T\left(\sigma_{e}\left(\left\{\sigma *_{i}\right\},\left\{x *_{i}\right\}\right)\right),
$$

where $T$ is the corresponding DT. This equation means also that $\sigma_{e}$ at the FP is a fixed point (or an eigenvector with the eigenvalue equal to 1 ) of $T$. In order to exclude a misunderstanding of different fixed points, we will call the fixed points of $T$ as its eigenvectors. As is known, the LF transformations $T$ can have only one or two eigenvectors in dependence on value of its discriminant. For $T$ from (8) one has for such eigenvectors $z_{1,2}$

$$
T\left(z_{1,2}\right)=z_{1,2}, \quad z_{1,2}=-i \frac{a-c}{d} \pm \sqrt{\frac{-(a-c)^{2}+4 b d}{4 d^{2}}}
$$

It follows from (13), that for $(a-c)^{2}<4 b d$ the corresponding $z_{1}$ will have a positive real part, what gives a physical conductivity. For $(a-c)^{2}>4 b d$ both eigenvectors have only imaginary part, i.e. they can correspond only to $\sigma$ with $\sigma_{d}=0, \sigma_{t} \neq 0$. In classical and usual systems this case is unphysical and cannot be realized (though, it can serve for a phenomenological approximate description of the low-temperature systems with quantum Hall effect [15]). It follows from (13) that one can always construct for a given physical $\sigma$ the transformation $T_{\sigma}$, which has $\sigma$ as its eigenvector,

$$
\sigma=\sigma_{d}+i \sigma_{t}, \quad 2 \sigma_{t}=-\left(\frac{a-c}{d}\right), \quad b / d=\sigma_{d}^{2}+\sigma_{t}^{2},
$$

As one can see from (14), this $\sigma$ does not define the corresponding $T_{\sigma}$ unambiguously, since there are 3 real parameters in $T$. The third parameter can be determined if $T$ has some additional constraint. There is a very important case of transformations $T$, satisfying the condition $T^{2}(z)=z$, (i.e. $T^{2} \sim I$ or $T^{-1} \sim T$, it includes all $T$ acting as permutations). For these transformations $a=-c$ and, consequently, one has

$$
\sigma_{t}=-a / d, \quad b / d=\sigma_{d}^{2}+\sigma_{t}^{2} .
$$

For transformations with $c=a$ and $b / d \geq 0$ the eigenvectors can be only real $z_{1,2}= \pm \sqrt{b / d}$.

Thus, we see that though a number of parameters of "dual" transformations $T$ becames larger (3 instead of 1 ), but, since a number of partial conductivities doubled, they are still not enough to make the DR essentially richer, than in a case with $H=0$ (netherless, some new possibilities appear, see below section 4). Fortunately, in any case one can define the transformation $T_{12}$, interchanging arbitrary $\sigma_{1}$ and $\sigma_{2}$,

$$
T_{12} \sigma_{1}=\sigma_{2}, \quad T_{12} \sigma_{2}=\sigma_{1}
$$


In components (16) has the form

$$
\begin{gathered}
\sigma_{2 d}=\frac{\bar{c} \sigma_{1 d}(\bar{a}+\bar{b} / \bar{c})}{\left(\sigma_{1 d}\right)^{2}+\left(\bar{a}+\sigma_{1 t}\right)^{2}}, \quad \sigma_{2 t}=\bar{c} \frac{\sigma_{1 d}^{2}+\left(\bar{a}+\sigma_{1 t}\right)\left(\sigma_{1 t}-b / c\right)}{\sigma_{1 d}^{2}+\left(\bar{a}+\sigma_{1 t}\right)^{2}} \\
\sigma_{1 d}=\frac{c \sigma_{2 d}(\bar{a}+b / c)}{\left(\sigma_{2 d}\right)^{2}+\left(\bar{a}+\sigma_{2 t}\right)^{2}}, \quad \sigma_{1 t}=\bar{c} \frac{\sigma_{2 d}^{2}+\left(\bar{a}+\sigma_{2 t}\right)\left(\sigma_{2 t}-b / c\right)}{\left(\sigma_{2 d}\right)^{2}+\left(\bar{a}+\sigma_{2 t}\right)^{2}} .
\end{gathered}
$$

Though, formally, this transformation relates 4 independent parameters and satisfies the condition $T^{2} \sim I$, it has one-parametric general solution of the form

$$
\begin{gathered}
a=-d \frac{\sigma_{1 d} \sigma_{2 t}+\sigma_{2 d} \sigma_{1 t}}{\sigma_{1 d}+\sigma_{2 d}}, \quad c=-a, \\
b=d \frac{\sigma_{1 d} \sigma_{2 t}^{2}+\sigma_{2 d} \sigma_{1 t}^{2}+\sigma_{1 d} \sigma_{2 d}\left(\sigma_{1 d}+\sigma_{2 d}\right)}{\sigma_{1 d}+\sigma_{2 d}},
\end{gathered}
$$

where $d$ is included as an arbitrary common factor. This fact reflects an existence of some hidden symmetry, wich effectively reduces a number of independent equations (see also discussions in the Sections 3 and 4).

\section{Fixed points and exact values of the effective conductivity.}

Having all mathematical machinery ready, let us consider the nontrivial fixed points of the DT. We begin with the known results for binary case $N=2$.

(1). A binary case $N=2$.

In this case the effective conductivity $\sigma_{e}$ depends on two sets of partial parameters $\sigma_{i d}, \sigma_{i t}, x_{i}, i=1,2$. As it was shown in the previous section, one can always find the DT $T_{12}$, interchanging partial conductivities of two phases. Then, using (11) and a symmetry of $\sigma_{e}$ one obtains the following DR

$$
\sigma_{e}\left(\sigma_{1}, x_{1} \mid \sigma_{2}, x_{2}\right)=T_{12}\left(\sigma_{e}\left(\sigma_{1}, x_{2} \mid \sigma_{2}, x_{1}\right)\right),
$$

At the point of equal phase concentrations one obtains the exact equation for $\sigma_{e(12)}$

$$
\sigma_{e}\left(\sigma_{1}, 1 / 2 \mid \sigma_{2}, 1 / 2\right)=T_{12}\left(\sigma_{e}\left(\sigma_{1}, 1 / 2 \mid \sigma_{2}, 1 / 2\right)\right),
$$

which means that this $\sigma_{e}$ is a fixed point (or an eigenvector) of $T_{12}$ transformation. From (15) and (18) one gets the exact value for $\sigma_{e}$

$$
\begin{gathered}
\sigma_{e d}\left(\sigma_{1 d}, 1 / 2 \mid \sigma_{2 d}, 1 / 2\right)=\sqrt{-\bar{a}^{2}+\bar{b}}=\sqrt{\sigma_{1 d} \sigma_{2 d}}\left(1+\left(\frac{\sigma_{1 t}-\sigma_{2 t}}{\sigma_{1 d}+\sigma_{2 d}}\right)^{2}\right)^{1 / 2}, \\
\sigma_{e t}\left(\sigma_{1}, 1 / 2 \mid \sigma_{2}, 1 / 2\right)=-\bar{a}=\frac{\sigma_{1 d} \sigma_{2 t}+\sigma_{2 d} \sigma_{1 t}}{\sigma_{1 d}+\sigma_{2 d}}
\end{gathered}
$$

which firstly has been obtained in this form in [13. It is easy to see that the change $\mathbf{H} \rightarrow-\mathbf{H}$ changes a sign of $\sigma_{e t}$ only, in accordance with the Onsager symmetry (3). The exact formulas (21) are very interesting result. It shows that the change of the exact result for $\sigma_{e d}$ at $H=0$ is completely determined 
by a difference of the partial transverse conductivities $\sigma_{1 t}-\sigma_{2 t}$. When they are equal, the diagonal part of the effective conductivity coincides (in its form) with that without magnetic field and the transverse part of $\sigma_{e}$ remains unchanged. Later it was shown that this property takes place for all concentrations, what allows to describe the systems with uniform transverse conductivity in terms of systems without a magnetic field [14. At the same time, when $\sigma_{1 d}=\sigma_{2 d}$, the effective transverse conductivity is simply equal to the average of partial transverse conductivities $\sigma_{e t}=\left(\sigma_{1 t}+\sigma_{2 t}\right) / 2$.

(2). 3 phase self-dual systems.

For 3-phase systems without magnetic field the FPs $(\{\sigma *\},\{x *\})$ of the DT exist, when two any phases have equal concentrations, for example, $x_{1}=$ $x_{2}=x$, (but otherwise they are arbitrary, except the normalization condition $\left.2 x+x_{3}=1\right)$ and their partial conductivities satisfy the condition [9, 10.

$$
x_{1}=x_{2}, \quad 2 x+x_{3}=1 ; \quad \sigma_{3}=\sqrt{\sigma_{1} \sigma_{2}} .
$$

One can try to find the FP of the same form for 3-phase systems with magnetic field. Then, the interchanging transformation $T_{12}$ can again be compensated by the permutation symmetry due to the equality $x_{1}=x_{2}$, and one obtains that the FP of this type can exist if

$$
T_{12} \sigma_{1}=\sigma_{2}, \quad T_{12} \sigma_{2}=\sigma_{1}, \quad T_{12} \sigma_{3}=\sigma_{3} .
$$

The last condition means that $\sigma_{3}$ must be also an eigenvector of $T_{12}$ transformation. It is possible only if $\sigma_{3}$ coincides with the effective conductivity for two phase systems at equal phase concentrations $\sigma_{e}^{(N=2)}$ from (21) (since this $\sigma_{e}^{(N=2)}$ is also the eigenvector of $\left.T_{12}\right)$

$$
\sigma_{3 d}=\sqrt{\sigma_{1 d} \sigma_{2 d}}\left(1+\left(\frac{\sigma_{1 t}-\sigma_{2 t}}{\sigma_{1 d}+\sigma_{2 d}}\right)^{2}\right)^{1 / 2}, \quad \sigma_{3 t}=\frac{\sigma_{1 d} \sigma_{2 t}+\sigma_{2 d} \sigma_{1 t}}{\sigma_{1 d}+\sigma_{2 d}} .
$$

The equalities (24) generalize the corresponding equalities (22) for systems with $H=0$ and reduce to them when $\sigma_{i t}=0, i=1,2,3$. Thus, we obtain that the effective conductivity of 3-phase self-dual systems for the set of partial parameters $(\{\sigma *\},\{x *\})$, satisfying $(24)$ and $x_{1}=x_{2}$, , is also the eigenvector of $T_{12}$ and, consequently, coincides with two phase effective conductivity from (21)

$$
\sigma_{e}^{(N=3)}(\{\sigma *\},\{x *\})=\sigma_{3}=\sigma_{e(12)}^{(N=2)} .
$$

The set of partial parameters, satisfying the FP conditions, has the same structure as the corresponding sets for 3-phase systems without magnetic field and generalizes it. In this case $\sigma_{e}$ does not depend on common concentration of two first phases and system behaves as a homogeneous one.

The similar FPs exist, when phase concentrations of other pairs of phases are equal:

$$
\text { 1) } \left.x_{1}=x_{3}, \quad \sigma_{2}=\sigma_{e(13)}^{(2)}, \quad 2\right) \quad x_{2}=x_{3}, \quad \sigma_{1}=\sigma_{e(23)}^{(2)},
$$


No any other nontrivial FPs appear. For example, a new FP can appear, when all concentrations are equal $x_{1}=x_{2}=x_{3}=1 / 3$. In this case, in principle, it is possible that the phases are permutated along the shemes (nontrivial cycles) $" 1 " \rightarrow " 2 " \rightarrow " 3 " \rightarrow " 1 "($ or $" 1 " \rightarrow " 3 " \rightarrow " 2 " \rightarrow$ "1"). It means that a transformation $T$ must exist such that

$$
T\left(\sigma_{1}\right)=\sigma_{2}, \quad T\left(\sigma_{2}\right)=\sigma_{3}, \quad T\left(\sigma_{3}\right)=\sigma_{1}, \quad T^{3} \sim I .
$$

Let us consider the corresponding transformations of the diagonal components

$$
\begin{gathered}
\sigma_{2 d}=\frac{\bar{c} \sigma_{1 d}(\bar{a}+b / c)}{\left(\sigma_{1 d}\right)^{2}+\left(\bar{a}+\sigma_{1 t}\right)^{2}}, \quad \sigma_{3 d}=\frac{\bar{c} \sigma_{2 d}(\bar{a}+b / c)}{\sigma_{2 d}^{2}+\left(\bar{a}+\sigma_{2 t}\right)^{2}}, \\
\sigma_{1 d}=\frac{\bar{c} \sigma_{3 d}(\bar{a}+b / c)}{\left(\sigma_{3 d}\right)^{2}+\left(\bar{a}+\sigma_{3 t}\right)^{2}} .
\end{gathered}
$$

It appears that the condition $T^{3} \sim I$ determines $T$ up to one parameter, say $a$

$$
a=c, \quad b / d=3 a^{2} / d^{2} .
$$

Then, one obtains from (28) for a 3 relations, which contain 6 parameters (partial conductivities). Another 3 independent relations can be obtained analogously from expressions for transverse components (they are more complicated, for this reason we do not write them here). As a results one has 6 various expressions for $a$. They give 15 nonlinear relations for 6 parameters. In principle, this overdetermined system of relations can have nontrivial solutions, even parametric, if there is some hidden symmetry, which reduces number of independent relations. Such situation usually takes place for exactly solvable models [17. If any solutions of this system of relations exist, they will give only diagonal eigenvectors, since, due to $\bar{a}=\bar{c}$, the corresponding eigenvectors of this $\mathrm{T}$ are $z_{1,2}= \pm \sqrt{3}|\bar{a}|$. This means that at this FP $\sigma_{e t}=0$. Such unusual result, though it seems an impossible one, needs a separate consideration.

\section{(3). General $N$-phase case}

We show now that for heterophase systems with arbitrary number $N$ of phases in magnetic field the duality relation (4) admits also the FPs, which are a generalization of the FP of the $N$-phase systems with $H=0$.

Of course, for systems with $N>3$, there are the fixed points with a structure similar to the $N=3$ case, when for some pair of phases $(i, j)$ their concentrations are equal $x_{i}=x_{j}$ and all other $\sigma_{l}(l \neq i, j)$ are equal between themselves and are equal to $\sigma_{e}$ of these two phases. But this case is trivial, since it reduces to the 3 -phase case. Another possibilities appear for $N>3$. It will be convenient to consider the cases with even and odd $N$ separately. Let us consider firstly a case when $N$ is even $N=2 M$. Then a new fixed point is possible, which corresponds to $M$ pairs of phases with equal concentrations and to the same transformation 
$T_{12}$ from (16). But now, $T_{12}$ must act as the interchanging operator on all $M$ pairs. For example, the fixed point with the phase concentrations

$$
x_{2 i-1}=x_{2 i}=y_{i}, \quad \sum y_{i}=1 / 2, \quad(i=1, \ldots, M)
$$

is possible, if

$$
T_{12} \sigma_{2 i-1}=\sigma_{2 i}, \quad T_{12} \sigma_{2 i}=\sigma_{2 i-1}, \quad(i=1, \ldots, M)
$$

For this one needs that all elements $a, b, c, d$ of $T_{12}$ must have the same dependence on the partial parameters of all pairs of phases

$$
\begin{gathered}
a=-d \frac{\sigma_{(2 i-1) d} \sigma_{2 i t}+\sigma_{2 i d} \sigma_{(2 i-1) t}}{\sigma_{(2 i-1) d}+\sigma_{2 i d}}, \quad c=-a, \\
b=d \frac{\sigma_{(2 i-1) d} \sigma_{2 i t}^{2}+\sigma_{2 i d} \sigma_{(2 i-1) t}^{2}+\sigma_{(2 i-1) d} \sigma_{2 i d}\left(\sigma_{(2 i-1) d}+\sigma_{2 i d}\right)}{\sigma_{(2 i-1) d}+\sigma_{2 i d}}, \quad i=1,2 \ldots M .
\end{gathered}
$$

Here partial conductivities of all phase pairs must be different. Then the equalities (32) define surfaces in the 4-dimensional spaces of partial parameters of the phase pairs. The effective conductivity $\sigma_{e}$ of $N$-phase system has in this case the exact value

$$
\sigma_{e}(\{\sigma\},\{x\})=\sigma_{e}^{(2)}=\sigma_{e i}^{(2)}, \quad(i=1, \ldots, M),
$$

where $\sigma_{e i}^{(2)}$ is the effective conductivity of the i-th pair, which all are equal. Note that again the equal concentrations of the phase pairs $y_{a}(a=1, \ldots, M)$ can be arbitrary, except the normalizing condition $\sum_{a=1}^{M} 2 y_{a}=1$. The exact value (31) does not depend on $y_{a}$ and ensures again the correct values for $\sigma_{e}$ in the limits $y_{a} \rightarrow 0,1(a=1, \ldots, M)$. Of course, the similar fixed points exist for other ways of the partition of $N$ phases into the pairs with equal concentrations. A number of such points is equal $\#_{2 M}=(2 M-1) !$ !. The exact values of $\sigma_{e}$ in these points have always the same general form

$$
\sigma_{e}^{(N)}(\{\sigma *\},\{x *\})=\sigma_{e(i j)}^{(2)} \quad(i<j, i, j=1, \ldots, N),
$$

where possible pairs $(i j)$ correspond to the phases with equal concentrations in the corresponding fixed points.

Now we consider a case when $N$ is odd $N=2 M+1$. In this case a new fixed point is possible if the above conditions of the even case (29) are supplemented by a condition on $\sigma_{2 M+1}$ analogous to the condition for $N=3$ case

$$
T_{12} \sigma_{2 M+1}=\sigma_{2 M+1}
$$

which ensures again the equality of $\sigma_{2 M+1}$ with $\sigma_{e}$ of the whole system. Thus, $\sigma_{e}$ is again equal to the exact value (21), coinciding with the exact value of system with $N=2 M$. Of course, the other fixed points related with various 
ways of choice of the phase pairs with equal concentrations are possible. Their number is equal to $\#_{2 M+1}=(2 M+1)$ !!. A general form of the exact values of $\sigma_{e}$ remains the same as in the even case with an additional equality

$$
\sigma_{e}^{(N)}(\{\sigma *\},\{x *\})=\sigma_{e(i j)}^{(2)}=\sigma_{k}, \quad i \neq j \neq k, \quad i<j \quad(i, j, k=1, \ldots, N) .
$$

where the pairs $i j$ correspond to the phases with equal concentrations and $k$ corresponds to the unpaired phases. Note that the structure of FPs is in an accordance with the symmetry.

It follows also from analysis of the case $N=3$ that the exact values of $\sigma_{e}$ at the point, where all phase concentrations are equal, are not universal, when partial conductivities have general values. An existence of new FP for $N>3$ connected with higher nontrivial cycles is also improbable, since all equations of the form $T^{n} \sim I,(n>3)$ require $a=c$ and the corresponding FPs can give only diagonal $\sigma_{e}$ (but they also need an additional consideration).

\section{Transformation between systems with $\mathbf{H} \neq 0$ and $\mathbf{H}=0$}

A more richer structure of the DT in systems with magnetic field allows also to construct various transformations, connecting effective conductivities of the initial system and other systems, related with the initial one. For example, the transformations, connecting effective conductivities of the systems in opposite magnetic fields, were constructed [12, 15]. Moreover, the transformation, connecting effective conductivities of two-phase systems with magnetic field and without it was constructed [16]. Strictly speaking, it connects the conductivities of systems with nonzero transverse component and systems with a pure diagonal structure. Such transformation allows to find the effective conductivity of inhomogeneous systems in a magnetic field, when it is known for these systems without a magnetic field. But, a derivation of this transformation in [16] was incomplete (in particular, it was not checked for known exact values (21)). Under its derivation two simplifying conjectures have been used: (1) the transformation, symmetrizing partial conductivities $\sigma_{1}$ and $\sigma_{2}$, will simultaneously symmetrize the effective conductivity too, (2) one parameter, say $c$, is effectively factorized and, since the conductivities "that differ only by a proportionality constant are obviously similar", can be taken equal to 1 . These conjectures correspond, instead of (11), to the following transformation of $\sigma_{e}$ (see also schematic diagrams of different transformations in fig.1)

$$
T_{m}\left(\sigma_{e}\left(\left\{\sigma_{i}\right\},\left\{x_{i}\right\}\right)\right)=\sigma_{e}\left(T_{m}\left(\left\{\sigma_{i}\right\}\right),\left\{x_{i}\right\}\right), \quad T_{m}(z)=\frac{z-i b^{\prime}}{-i z+a} .
$$

Since it differs from (11), we will construct an analogous transformation in an accordance with the transformation rules (11) (fig.1b), taking into account all 3 parameters. The parameters of such transformation (let us call it $T_{h}$ ) must satisfy two following equations, denoting a cancellation of imaginary parts of $\sigma_{i}^{\prime}$ (i.e. $\operatorname{Im} T\left(\sigma_{i}\right)=0$ )

$$
\sigma_{i t}^{\prime}=\bar{c} \frac{\sigma_{i d}^{2}+\left(\bar{a}+\sigma_{i t}\right)\left(\sigma_{i t}-b / c\right)}{\left(\sigma_{i d}\right)^{2}+\left(\bar{a}+\sigma_{i t}\right)^{2}}=0,(i=1,2) .
$$




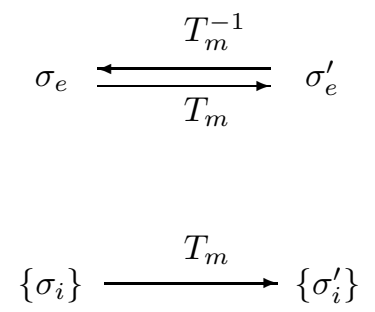

(a)

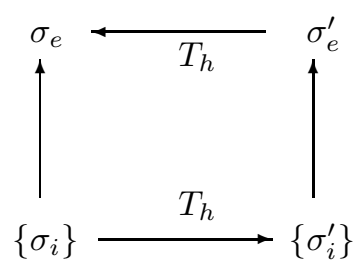

(b)

Fig.1. (a) A schematic diagram of transformations $T_{m}$ used in [16], (b) a schematic diagram of transformation $T_{h}$ used in this paper.

Note, that this transformation is not equivalent to a usual diagonalization of matrices $\hat{\sigma}_{i}$, since they, due to their antisymmetric parts, cannot have real eigenvalues. Two equations (36) are not enough for a complete determination of $T_{h}$ (i.e. the parameters $\bar{a}_{h}, \bar{b}_{h}, \bar{c}_{h}$, for brevity, we will not write this additional index $h$ and bars in this section) in terms of partial conductivities of the initial system $\sigma_{i d}, \sigma_{i t}$. The equations (36) permit the determination of the parameter $a$ and the ratio $b^{\prime}=b / c$

$$
\begin{gathered}
a_{ \pm}=\frac{\left|\sigma_{2}\right|^{2}-\left|\sigma_{1}\right|^{2} \pm \sqrt{B}}{2\left(\sigma_{1 t}-\sigma_{2 t}\right)}, \quad b_{ \pm}^{\prime}=\frac{\left|\sigma_{1}\right|^{2}-\left|\sigma_{2}\right|^{2} \pm \sqrt{B}}{2\left(\sigma_{1 t}-\sigma_{2 t}\right)} \\
B=\left[\left(\sigma_{1 t}-\sigma_{2 t}\right)^{2}+\left(\sigma_{1 d}-\sigma_{2 d}\right)^{2}\right]\left[\left(\sigma_{1 t}-\sigma_{2 t}\right)^{2}+\left(\sigma_{1 d}+\sigma_{2 d}\right)^{2}\right]
\end{gathered}
$$

where, evidently, $B \geq 0$. It follows from (34) that their denominators $D_{i}$ can be represented in the factorised form

$$
D_{i}=\sigma_{i d}^{2}+\left(a+\sigma_{i t}\right)^{2}=\left(a+\sigma_{i t}\right)\left(a+b^{\prime}\right), \quad i=1,2 .
$$

Then, the diagonal (or real) parts of $\sigma_{i}$ transform under $T_{h}$ as

$$
\sigma_{i d}^{\prime}=\sigma_{i d} \frac{c\left(a+b^{\prime}\right)}{\left(\sigma_{i d}\right)^{2}+\left(a+\sigma_{i t}\right)^{2}}=\frac{c \sigma_{i d}}{\sigma_{a i}}, \quad \sigma_{a i}=a+\sigma_{i t} .
$$

The primed diagonal components are determined up to a common factor $c$ (it was assumed to be 1 in [16.) In order to find $c$ (or $b$ ) one needs one additional relation on parameters $a, b, c$. Since the transformation $T_{h}$ does not depend on phase concentrations, it is convenient to choose an additional relation by requiring that $T_{h}$ must reproduce one of the two exact expressions for components of $\sigma_{e}$ at equal phase concentrations $x_{1}=x_{2}=1 / 2$ from (21). For example, the requirement of a reproduction of $\sigma_{e d}$ with a usage of the diagonal components $\sigma_{i d}^{\prime}$ from (39) and the exact expression for $\sigma_{e}^{\prime}=\sqrt{\sigma_{1 d}^{\prime} \sigma_{2 d}^{\prime}}$ at $x_{1}=x_{2}=1 / 2$ gives the desired additional relation

$$
A=\left[1+\left(\frac{\sigma_{1 t}-\sigma_{2 t}}{\sigma_{1 d}+\sigma_{2 d}}\right)^{2}\right]^{1 / 2}=\frac{\left(a+b^{\prime}\right)\left(\sigma_{a 1} \sigma_{a 2}\right)^{1 / 2}}{\sigma_{1 d} \sigma_{2 d}+(a / c)^{2} \sigma_{a 1} \sigma_{a 2}}
$$




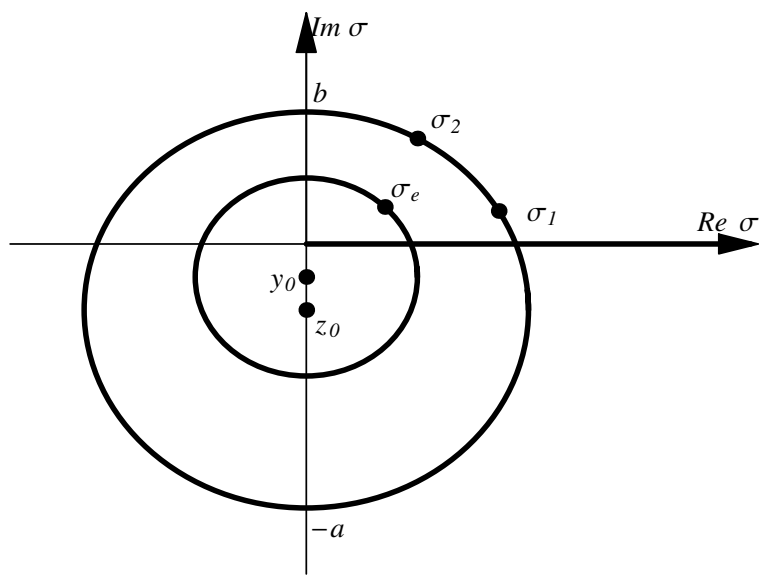

Fig.2. A schematic picture of two circles on the complex conductivity plane, appearing under transformation $T_{h}$ used in this paper.

Using the relation (40) one can determine only $|c|$ through $A$ and $\sigma_{a i}$, which depend on partial conductivities $\sigma_{i d}, \sigma_{i t}$,

$$
c^{2}=\frac{a^{2} A \sigma_{a 1} \sigma_{a 2}}{\left(a+b^{\prime}\right)\left(\sigma_{a 1} \sigma_{a 2}\right)^{1 / 2}-\sigma_{1 d} \sigma_{2 d} A} .
$$

In order to find a sign of $c$ and to check the self-consistency of the transformation $T_{h}$ one must reproduce the exact expression for the transverse component $\sigma_{e t}$ (and vice versa, if the requirement of a reproduction of $\sigma_{e t}$ is chosen as a first one). This means that the following relation must hold

$$
\frac{\sigma_{1 d} \sigma_{2 t}+\sigma_{2 d} \sigma_{1 t}}{\sigma_{1 d}+\sigma_{2 d}}=c \frac{{\sigma_{e}^{\prime}}^{2}-a b^{\prime}}{{\sigma_{e}^{\prime}}^{2}+a^{2}}=c \frac{\sigma_{1 d} \sigma_{2 d}-\left(a b^{\prime} / c^{2}\right) \sigma_{a 1} \sigma_{a 2}}{\sigma_{1 d} \sigma_{2 d}+(a / c)^{2} \sigma_{a 1} \sigma_{a 2}} .
$$

The relations (40)-(42) give us a highly nontrivial check of a self-consistency of the derived transformation. The direct check of them is a rather complicated task. Fortunately, there is more simple and elegant way to see this, which can make the derived formulas more understandable.

Let us consider the action of the transformation $T_{h}$ in the complex plane $z$ in more details. By construction, it maps the given pair of complex partial conductivities $\sigma_{1}, \sigma_{2}$, into the real axis. According to general properties of the LFT, $T_{h}$ must map into the real axis a whole circumference, containing partial conductivities $\sigma_{1}, \sigma_{2}$ (see fig.2).

This fact was noted in [16]. It allows to determine a position of the center of this circumference, which is situated on the imaginary axis in the point $z_{0}$, and its radius $R$

$$
z_{0}=\left(b^{\prime}-a\right) / 2=\frac{\left|\sigma_{1}\right|^{2}-\left|\sigma_{2}\right|^{2}}{2\left(\sigma_{1 t}-\sigma_{2 t}\right)}, \quad R=\left|a+b^{\prime}\right| / 2=\frac{\sqrt{B}}{2\left|\sigma_{1 t}-\sigma_{2 t}\right|} .
$$


At the same time $T_{h}$ maps the real axis into another circumference described by the equation

$$
x^{2}+\left(y-y_{0}\right)^{2}=\frac{c^{2} R^{2}}{a^{2}}, \quad y_{0}=\frac{c\left(a-b^{\prime}\right)}{2 a}=-\frac{c z_{0}}{a} .
$$

Just into this circumference $T_{h}$ maps $\sigma_{e}^{\prime}$. Using the explicit formulas for $\sigma_{e}$ from (21), one can check that $\sigma_{e}$ belongs to the first circumference. In order for $T_{h}\left(\sigma_{e}^{\prime}\right)=\sigma_{e}$, these two circumference must coincide! This gives a more simple expressions for $c$ and $b$

$$
c=-a, \quad b=b^{\prime} c=-a b^{\prime} .
$$

The equality $c=-a$ means also that effectively $T_{h}^{2} \sim I$, since it transforms the first circle into itself. Substituting this parameters in (39) one obtains the explicit expressions for $T_{h}$ and $\sigma_{i d}^{\prime}$. One can check also that the relations (40)-(42) are fulfilled (for both $a_{ \pm}$). It is interesting to compare the inverse transformation $T_{m}^{-1}$, found in [16] under conjecture $c=1$, with $T_{h}$ with $c=-a$, when they act on their primed conductivities, belonging to the real axes $y=0$ (it is obvious that they differ in general),

$$
\begin{gathered}
T_{m}^{-1}(z)=\frac{a z+i b^{\prime}}{i z+1} \underset{y=0}{\longrightarrow} \frac{\left(a+b^{\prime}\right) x+i\left(-a x^{2}+b^{\prime}\right)}{1+x^{2}}, \\
T_{h}(z)=\frac{a z+i b^{\prime}}{i z-a} \underset{y=0, x=-a \bar{x}}{\longrightarrow} \frac{\left(a+b^{\prime}\right) \bar{x}-i\left(a \bar{x}^{2}-b^{\prime}\right)}{1+\bar{x}^{2}},
\end{gathered}
$$

where we have used the fact that the primed values for $T_{h}$ contain a common factor $c=-a$, i.e. $x=-a \bar{x}$. One can see that in this case they luckily coincide! Since $T_{h}\left(\sigma_{e}^{\prime}\right)$ belongs at $c=-a$ to the first circumference, this automatically ensures a fulfillment of the second requirement. All equalities necessary for fulfillment of (39) correspond to the known equalities between lengths of various chords, their projections on a diameter and the radius of the circles (see fig.3.). For example, the equality (37) takes the form

$$
A=\frac{2 R \sigma_{a}}{\sigma_{a}^{2}+\sigma_{1 d} \sigma_{2 d}}=1 /\left(1-\delta / 2 R \sigma_{a}\right), \quad \sigma_{a}^{2}=\sigma_{a 1} \sigma_{a 2},
$$

where $\sigma_{a}^{2}$ is a squared geometrical average of $a+\sigma_{i},(i=1,2)$, and $\delta$ defines a difference between $\sigma_{x}^{2}=2 R \sigma_{a}-\sigma_{a}^{2}$, a squared real projection of the chord, having an imaginary projection $\sigma_{a}$, and a squared geometric average of $\sigma_{i d}$

$$
\delta=\sigma_{x}^{2}-\sigma_{1 d} \sigma_{2 d}
$$

It is worth to note that the transformation $T_{h}$ with $c=a$ transforms initial system into that with an opposite magnetic field $-\mathbf{H}$.

Thus, the obtained full (3-parametric) transformation $T_{h}$ allows to find the effective conductivity of binary inhomogeneous system in magnetic field at arbitrary phase concentrations in an explicit form, if the partial conductivities 


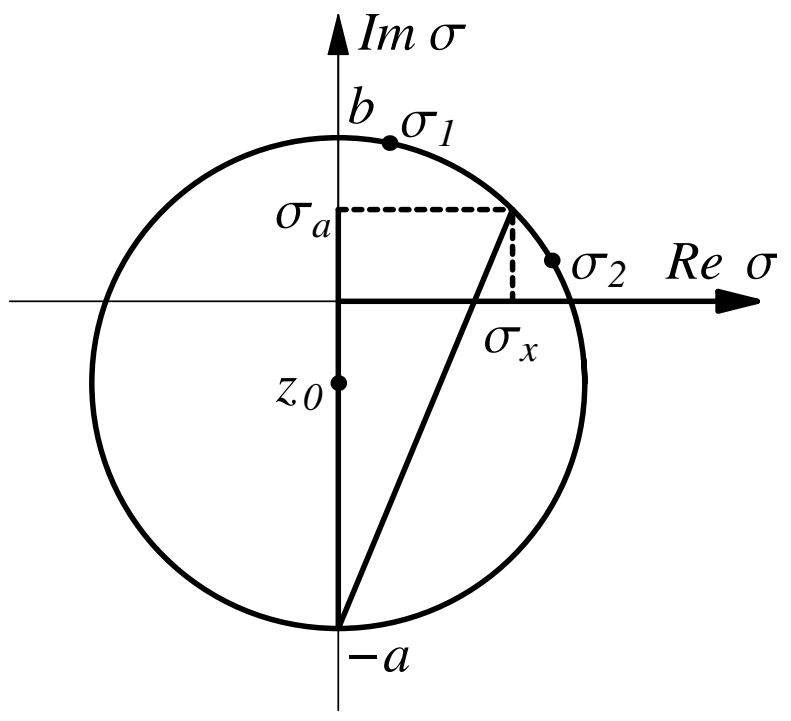

Fig.3. A schematic picture of the chord, defining a geometrical sense of the factor $A$ from the expression for the real part of the exact $\sigma_{e}$.

of the initial system in magnetic field $\sigma_{i d}, \sigma_{i t}$, and the effective conductivity of artificial system at arbitrary phase concentrations are known. This question together with other methods of construction of explicit expressions for $\sigma_{e}$ in magnetic field at arbitrary phase concentrations and their high magnetic field behaviour will be considered in detail in the forthcoming paper.

\section{$\underline{\text { 5. Conclusion }}$}

Using the exact duality transformations and symmetry properties of $2 \mathrm{D}$ isotropic heterophase systems we have found all possible physical fixed points (except possible very special FPs with unusual properties) and the corresponding exact values of their effective conductivities in magnetic field. The obtained results take place for various heterophase systems (regular and nonregular as well as random), satisfying the symmetry and self-duality conditions, and show very interesting properties. As in the case $N>2, H=0$, an existence of hyperplanes in the concentration space with constant $\sigma_{e d, e t}$, when partial conductivities belong to some surfaces in their space, is unusual. These results admit a direct experimental checking. The 3-parametric transformation, connecting effective conductivities of two-phase systems with magnetic field and without it, was also constructed. It allows to find effective conductivity of two-phase systems in a perpendicular magnetic field at arbitrary phase concentrations, if the effective conductivity of these systems at $H=0$ is known.

Acknowledgments 
The authors are thankful to Prof.A.P.Veselov for very useful discussions of some mathematical questions. This work was supported by the RFBR grants 00-15-96579, 02-02-16403, and by the Royal Society grant 2004/R4-EF.

\section{References}

[1] L.D.Landau, E.M.Lifshitz, Electrodynamics of condensed media, Moscow, 1982 (in Russian).

[2] G.Allodi et al., Phys.Rev. B56 (1997) 6036; M.Hennion et al., Phys.Rev.Lett. 81 (1998) 1957; Y.Moritomo et al., Phys.Rev. B60 (1999) 9220 .

[3] R.Xu et al., Nature 390 (1997) 57.

[4] S.Kirkpatrick, Rev.Mod.Phys. 45 (1973) 574.

[5] S.A.Bulgadaev, "Conductivity of 2D random heterophase systems", cond-mat/0410073 (2004), to be published.

[6] D.Stroud, D.J.Bergmann, Phys.Rev.B62 (2000) 6603.

[7] Yu.A.Dreizin, A.M.Dykhne ZhETF 63 (1972) 242 (Sov.Phys. JETP 36 (1973) 127); I.M.Kaganova, M.I.Kaganov, cond-mat/0402426 (2004).

[8] J.B.Keller, J.Math.Phys., 5 (1964) 548.

[9] A.M.Dykhne, ZhETF 59 (1970) 110 (JETP 32 (1970) 63).

[10] S.A.Bulgadaev, Phys.Lett. A313 (2003) 144.

[11] S.A.Bulgadaev, Europhys.Lett.64 (2003) 482, Phys.Lett. A313 (2003) 106, cond-mat/0410058 to be published.

[12] A.M.Dykhne, ZhETF 59 (1970) 641 (JETP 32 (1970) 348).

[13] B.I.Shklovskii, ZhETF 72 (1977) 288; B.Ya.Balagurov, ZhETF 82 (1982) 1333.

[14] D.J.Bergmann, D.G.Stroud, Phys.Rev.B30 (1984) 447.

[15] A.M.Dykhne, I.M.Ruzin, Phys.Rev.50 (1994) 2369; V.G.Marikhin, Pis'ma v ZhETF, 71 (2000) 391.

[16] G.W.Milton, Phys.Rev. B38 (1988) 11296.

[17] R.J.Baxter, Exactly solved models of statistical mechanics, Academic Press (1982). 